FACTA UNIVERSITATIS (NIŠ)

Ser. Math. Inform. Vol. 35, No 2 (2020), 357-366

https://doi.org/10.22190/FUMI2002357M

\title{
LEGENDRE CURVES ON 3-DIMENSIONAL f-KENMOTSU MANIFOLDS ADMITTING SCHOUTEN-VAN KAMPEN CONNECTION
}

\begin{abstract}
Ashis Mondal
(C) 2020 by University of Niš, Serbia | Creative Commons Licence: CC BY-NC-ND

Abstract. In the present paper, biharmonic Legendre curves with respect to SchoutenVan Kampen connection have been studied on three-dimensional f-Kenmotsu manifolds. Locally $\phi$-symmetric Legendre curves on three-dimensional f-Kenmotsu manifolds with respect to Schouten-Van Kampen Connection have been introduced. Also, slant curves have been studied on three-dimensional f-Kenmotsu manifolds with respect to SchoutenVan Kampen connection. Finally, we have constructed an example of a Legendre curve in a 3-dimensional f-Kenmotsu manifold.

Keywords: Legendre curves; f-Kenmotsu manifold; Locally $\phi$-symmetric Legendre curves; Schouten-Van Kampen connection; Slant curve.
\end{abstract}

\section{Introduction}

In the study of contact manifolds, Legendre curves play an important role, e.g., a diffeomorphism of a contact manifold is a contact transformation if and only if it maps Legendre curves to Legendre curves. Legendre curves on contact manifolds have been studied by C. Baikoussis and D. E. Blair in the paper [2]. Originally, the notion of Legendre curve was defined for curves in a contact three-manifolds with the help of a contact form. This notion of Legendre curves can be also extended to almost contact manifolds [22]. Curves satisfying the properties of Legendre curves in almost contact metric manifolds are known as almost contact curves [5]. In [16], A. Sarkar, S. K. Hui and M. Sen have studied Legendre curves on three dimensional trans-Saskian manifold. J. Welyzcko [22], studied Legendre curves on a three-dimensional trans-Sasakian manifolds with respect to Levi-Civita connections. In [5], the authors have introduced a 1-parameter family of linear connections on three-dimensional almost contact metric manifolds to study biharmonic curves on almost contact manifolds. The author has studied some curves on three-dimensional

Received January 21, 2018; accepted May 03, 2020

2010 Mathematics Subject Classification. Primary 53C15; Secondary 53C25, 
trans-Sasakian manifolds with semi-symmetric metric connection [14]. The author of the present paper has also studied some curves on $\alpha$-Sasakian manifolds with indefinite metric [15]. The Schouten-Van Kampen connection is one of the most natural connections adapted to a pair of complementary distributions on a differentiable manifold endowed with an affine connection [3],[6], [17]. Solov'es investigated hyperdistributions in Riemannian manifolds using Schouten-Van Kampen connection [18]. Then Olszak studied the Schouten-Van Kampen connection to an almost contact metric structure [13]. He characterized some classes of almost contact metric manifolds with Schouten-Van Kampen connection and found certain curvature properties of this connection of these manifolds. In the present paper, we are interested to study biharmonic Legendre curves with respect to Schouten-Van Kampen connection on a three-dimensional f-Kenmotsu manifold. We also introduce locally $\phi$-symmetric almost contact curves with respect to Schouten-Van Kampen connections on a three-dimensional f-Kenmotsu manifold.

The present paper is organized as follows: After the introduction, we have given some required preliminaries in Section 2. In Section 3, we have studied biharmonic Legendre curves with respect to Schoute-Van Kampen connection. In Section 4, we have considered locally $\phi$-symmetric Legendre curves on three-dimensional fKenmotsu manifolds with respect to Schouten-Van Kampen connection. In Section 5 , we have studied slant curves with respect to Schouten-Van Kampen connection. In the last section, we have constructed an example of Legendre curve in a threedimensional f-Kenmotsu manifold.

\section{Preliminaries}

Let $M$ be a connected almost contact metric manifold with an almost contact metric structure $(\phi, \xi, \eta, g)$, that is, $\phi$ is an $(1,1)$ tensor field, $\xi$ is a vector field, $\eta$ is an 1 -form and $g$ is compatible Riemannian metric such that

$$
\begin{gathered}
\phi^{2} X=-X+\eta(X) \xi, \quad \eta(\xi)=1, \quad \phi \xi=0, \quad \eta \phi=0, \\
g(\phi X, \phi Y)=g(X, Y)-\eta(X) \eta(Y), \\
g(X, \phi Y)=-g(\phi X, Y), \quad g(X, \xi)=\eta(X),
\end{gathered}
$$

for all $X, Y \in T(M)[1]$. The fundamental 2-form $\Phi$ of the manifold is defined by

$$
\Phi(X, Y)=g(X, \phi Y),
$$

for $X, Y \in T(M)$. An almost contact metric manifold is normal if $[\phi, \phi](X, Y)+$ $2 d \eta(X, Y) \xi=0$.

An almost contact metric structure $(\phi, \xi, \eta, g)$ on a manifold $M$ is called $\mathrm{f}$ Kenmotsu manifold if this may be expressed by the condition [11]

$$
\left(\nabla_{X} \phi\right) Y=f\{g(\phi X, Y) \xi-\eta(Y) \phi X\},
$$


where $f \in C^{\infty}(M)$ such that $d f \wedge \eta=0$ and $\nabla$ is Levi-Civita connection on $M$. If $f=\alpha=$ constant $\neq 0$, then the manifold is an $\alpha$-Kenmotsu manifold [7]. 1Kenmotsu manifold is a Kenmotsu [8]. If $\mathrm{f}=0$, then the manifold is cosymplectic [7]. An f-Kenmotsu manifold is said to be to be regular if $f^{2}+f^{\prime} \neq 0$, where $f^{\prime}=\xi(f)$.

For an f-Kenmotsu manifold it follows that

$$
\nabla_{X} \xi=f\{X-\eta(X) \xi\}
$$

Then using (2.6), we have

$$
\left(\nabla_{X} \eta\right) Y=f(g(X, Y)-\eta(X) \eta(Y)) .
$$

The condition $d f \wedge \eta=0$ holds if $\operatorname{dim} \mathrm{M} \geqslant 5$. This does not hold in general if dim $\mathrm{M}=3$ [12]. In a 3-dimensional f-Kenmotsu manifold $\mathrm{M}$, we have [12]

$$
\begin{aligned}
& R(X, Y) Z=\left(\frac{r}{2}+2 f^{2}+2 f^{\prime}\right)\{g(Y, Z) X-g(X, Z) Y\} \\
&-\left(\frac{r}{2}+3 f^{2}+3 f^{\prime}\right)\{g(Y, Z) \eta(X) \xi-g(X, Z) \eta(Y) \xi \\
&+\eta(Y) \eta(Z) X-\eta(X) \eta(Z) Y\} \\
& S(X, Y)=\left(\frac{r}{2}+f^{2}+f^{\prime}\right) g(X, Y)-\left(\frac{r}{2}+3 f^{2}+3 f^{\prime}\right) \eta(X) \eta(Y), \\
& Q X=\left(\frac{r}{2}+f^{2}+f^{\prime}\right) X-\left(\frac{r}{2}+3 f^{2}+3 f^{\prime}\right) \eta(X) \xi,
\end{aligned}
$$

where $\mathrm{R}$ denotes the curvature tensor, $S$ is the Ricci tensor of type $(0,2), \mathrm{Q}$ is the Ricci operator and $r$ is the scalar curvature of the manifold $M$.

The Schouten-Van Kampen connections [9], [13] $\tilde{\nabla}$ and the Levi-Civita connection $\nabla$ are related by

$$
\tilde{\nabla}_{X} Y=\nabla_{X} Y-\eta(Y) \nabla_{X} \xi+\left(\nabla_{X} \eta\right)(Y) \xi
$$

for all vector fields $X, Y$ on $M$. With the help of (2.6) and (2.7), the above equation takes the form

$$
\tilde{\nabla}_{X} Y=\nabla_{X} Y+f\{g(X, Y) \xi-\eta(Y) X\},
$$

for an f-Kenmotsu manifold. So, we obtain the following Proposition by using (2.1)-(2.6) and (2.10):

Proposition 2.1. The Schouten-Van Kampen connections $\tilde{\nabla}$ on f-Kenmotsu manifold, we have the following properties $\tilde{\nabla} g=0, \tilde{\nabla} \eta=0$ and $\tilde{\nabla} \xi=0$. Also from (2.10), the tensor $\tilde{T}$ of the Schouten-Van Kampen connection is [21]

$$
\tilde{T}(X, Y)=\eta(X) \nabla_{Y} \xi-\eta(Y) \nabla_{X} \xi+2 d \eta(X, Y) \xi,
$$


for all fields X, Y on M.

Let $\tilde{\nabla}_{\dot{\gamma}}$ denote the covariant differentiation along $\gamma$ with respect to SchoutenVan Kampen connection on $M$. We shall say that $\gamma$ is a Frenet curve with respect to Schouten-Van Kampen connection if one of the following three cases holds:

(a) $\gamma$ is of osculating order 1, i.e., $\tilde{\nabla}_{t} t=0$ (geodesic).

(b) $\gamma$ is of osculating order 2, i.e., there exist two orthonormal vector fields $t(=\dot{\gamma}), n$ and a non-negative function $\tilde{k}$ (curvature) along $\gamma$ such that $\tilde{\nabla}_{t} t=\tilde{k} n$, $\tilde{\nabla}_{t} n=-\tilde{k} t$.

(c) $\gamma$ is of osculating order 3 , i.e., there exist three orthonormal vectors $t(=\dot{\gamma})$, $n, b$ and two non-negative functions $\tilde{k}$ (curvature) and $\tilde{\tau}$ (torsion) along $\gamma$ such that

$$
\begin{gathered}
\tilde{\nabla}_{t} t=\tilde{k} n, \\
\tilde{\nabla}_{t} n=-\tilde{k} t+\tilde{\tau} b, \\
\tilde{\nabla}_{t} b=-\tilde{\tau} n .
\end{gathered}
$$

The vector fields $t$ and $n$ along $\gamma$ in the above equations are related by $n=\phi t$ and hence $b=\xi$ along $\gamma$. With respect to Schouten-Van Kampen connection, a Frenet curve of osculating order 3 for which $\tilde{k}$ is a positive constant and $\tilde{\tau}=0$ is called a circle in $M$; a Frenet curve of osculating order 3 is called a helix in $M$ if $\tilde{k}$ and $\tilde{\tau}$ both are positive constants and the curve is called a generalized helix with respect to Schouten-Van Kampen connection if $\frac{\tilde{k}}{\tilde{\tau}}$ is a constant.

A Frenet curve $\gamma$ in an almost contact metric manifold is said to be almost contact curve if it is an integral curve of the distribution $\mathcal{D}=$ ker $\eta$. Formally, it is also said that a Frenet curve $\gamma$ in an almost contact metric manifold is an almost contact curve if and only if $\eta(\dot{\gamma})=0$ and $g(\dot{\gamma}, \dot{\gamma})=1$. For more details we refer $[2],[4],[10],[22]$. It is to be mentioned that in the paper [5], curves satisfying the above properties on almost contact manifolds have been termed as almost contact curve while Welyczko [22] has termed such curves on almost contact manifolds as Legendre curves. Henceforth, by Legendre curves on almost contact manifolds we shall mean almost contact curves.

A Frenet curve is called a slant curve if it makes a constant angle with Reeb vector field $\xi$. If a curve $\gamma$ on an almost contact metric manifold is a slant curve then $\eta(\dot{\gamma})=\cos \theta$ and $g(\dot{\gamma}, \dot{\gamma})=1$ where $\theta$ is a constant and is called slant angle. In particular if the angle is $\frac{\pi}{2}$, the curve becomes a Legendre curve.

\section{Biharmonic almost contact curves with respect to Schouten-Van Kampen connection}

In this section we study biharmonic almost contact curves on a three-dimensional f-Kenmotsu manifold with respect to Schouten-Van Kampen connection.

Definition 3.1. A Legendre curve $\gamma$ on a three-dimensional f-Kenmotsu manifold is called biharmonic with respect to Schouten-Van Kampen connection if it 
satisfies the equation [5]

$$
\tilde{\nabla}_{t}^{3} t+\tilde{\nabla}_{t} \tilde{T}\left(\tilde{\nabla}_{t} t, t\right)+\tilde{R}\left(\tilde{\nabla}_{t} t, t\right) t=0,
$$

where $\tilde{T}$ is torsion of the Schouten-Van Kampen connection and $\dot{\gamma}=t$ is tangent vector field of the curve.

Let us consider a Legendre curve $\gamma$ with respect to Schouten-Van Kampen connection on a three-dimensional f-Kenmotsu manifold. We take $\{t, \phi t, \xi\}$ as right handed system when $\phi t=-n, \phi n=t$.

Let $\tilde{R}$ and $\mathrm{R}$ be the Riemannian curvature tensor with respect to Schouten-Van Kampen connection and Levi-civita connection respectively. Then the relation between $\tilde{R}$ and $\mathrm{R}$ is given by [9]

$$
\begin{array}{ll}
\tilde{R}(X, Y) Z=R(X, Y) Z+f^{2}\{g(Y, Z) X-g(X, Z) Y\} \\
3.2) & +f^{\prime}\{g(Y, Z) \eta(X) \xi-g(X, Z) \eta(Y) \xi+\eta(Y) \eta(Z) X-\eta(X) \eta(Z) Y\} .
\end{array}
$$

For a Legendre curve $\eta(t)=0, \eta(n)=0$ because we have considered Frenet frame as $\{t, \phi t, \xi\}$ as a right handed system when $\phi t=-n, \phi n=t$. Using this facts in (3.2) and considering (2.8), we get

$$
\tilde{R}(n, t) t=\left(\frac{r}{2}+3 f^{2}+2 f^{\prime}\right) n .
$$

By Frenet formula (2.15), (2.16), (2.17) and (2.18) we get

$$
\tilde{\nabla}_{t}^{3} t=-3 \tilde{k} \tilde{k}^{\prime} t+\left(\tilde{k}^{\prime \prime}-\tilde{k}^{3}-\tilde{\kappa} \tilde{\tau}^{3}\right) n+\left(2 \tilde{\tau} \tilde{\kappa}^{\prime}+\tilde{\kappa} \tilde{\tau}^{\prime}\right) b,
$$

where $n=-\phi t, b=\xi$. In view of $(2.13)$

$$
\tilde{\nabla}_{t} \tilde{T}\left(\tilde{\nabla}_{t} t, t\right)=0 .
$$

By virtue of (3.3), (3.4) and (3.5) we get

$$
\begin{aligned}
\tilde{\nabla}_{t}^{3} t+\tilde{k} \tilde{R}(n, t) t & =-3 \tilde{k} \tilde{k}^{\prime} t+\left\{\tilde{k}^{\prime \prime}-\tilde{k}^{3}-\tilde{\kappa} \tilde{\tau}^{2}+\tilde{k}\left(\frac{r}{2}+3 f^{2}+2 f^{\prime}\right)\right\} n \\
& +\left(2 \tilde{\tau} \tilde{\kappa}^{\prime}+\tilde{\kappa} \tilde{\tau}^{\prime}\right) b .
\end{aligned}
$$

By virtue of (3.1) and observing the components of the right hand side of (3.6), we get $\tilde{\kappa}$ and $\tilde{\tau}$ are constant such that $\tilde{\kappa}^{2}+\tilde{\tau}^{2}=\frac{r}{2}+3 f^{2}+2 f^{\prime}$. Hence we can state the following theorem

Theorem 3.1. Let $\gamma$ be a non-geodesic Legendre curve with respect to SchoutenVan Kampen connection on three-dimensional f-Kenmotsu manifold. Then the Legendre curve is a helix with respect to the Schouten-Van Kampen connection such that $\tilde{\kappa}^{2}+\tilde{\tau}^{2}=\frac{r}{2}+3 f^{2}+2 f^{\prime}$. The converse statement is true if the torsion tensor is constant along $\gamma$. 


\section{Locally $\phi$-symmetric Legendre curves with respect to Schouten-Van Kampen connection}

The notion of locally $\phi$-symmetric manifolds was introduced by T. Takahashi [19] in the context of Sasakian geometry. Since every smooth curve is a one-dimensional differentiable manifold, we may apply the concept of local $\phi$-symmetry on a smooth curve. In [14], locally $\phi$-symmetric Legendre curves have been studied.

Definition 4.1. With respect to Schouten-Van Kampen connection a threedimensional f-Kenmotsu manifold will be called locally $\phi$-symmetric if it satisfies

$$
\phi^{2}\left(\tilde{\nabla}_{W} \tilde{R}\right)(X, Y) Z=0,
$$

for all vector fields $X, Y, Z, W$ orthogonal to $\xi$.

Definition 4.2. With respect to Schoutn-Van Kampen connection a Legendre curve $\gamma$ on a three-dimensional $f$-Kenmotsu manifold will be called locally $\phi$ symmetric if it satisfies

$$
\phi^{2}\left(\tilde{\nabla}_{t} \tilde{R}\right)\left(\tilde{\nabla}_{t} t, t\right) t=0,
$$

where $t=\dot{\gamma}$. Here we shall establish the following:

Theorem 4.1. A necessary and sufficient condition for a non-geodesic Legendre curve on a three-dimensional f-Kenmotsu manifold with constant structure function to be locally $\phi$-symmetric with respect to the Schouten-Van Kampen connection is $r=-6 f^{2}$, where $\mathrm{r}$ is the scalar curvature of the manifold with respect to Levi-Civita connection.

By definition of covariant differentiation of the Riemannian curvature tensor $R$ of type $(1,3)$ we obtain

$$
\left(\tilde{\nabla}_{t} \tilde{R}\right)\left(\tilde{\nabla}_{t} t, t\right) t=\tilde{\nabla}_{t} \tilde{R}\left(\tilde{\nabla}_{t} t, t\right) t-\tilde{R}\left(\tilde{\nabla}_{t}^{2} t, t\right) t-\tilde{R}\left(\tilde{\nabla}_{t} t, \tilde{\nabla}_{t} t\right) t-\tilde{R}\left(\tilde{\nabla}_{t} t, t\right) \tilde{\nabla}_{t} t .
$$

Using Serret-Frenet formula, from the above equation we get

$$
\begin{aligned}
\left(\tilde{\nabla}_{t} \tilde{R}\right)\left(\tilde{\nabla}_{t} t, t\right) t & =\tilde{\nabla}_{t} \tilde{R}\left(\tilde{\nabla}_{t} t, t\right) t+\tilde{k}^{2} \tilde{R}(t, t) t-\tilde{k} \tilde{\tau} \tilde{R}(b, t) t \\
& -\tilde{k}^{\prime} \tilde{R}(n, t) t-\tilde{k}^{2} \tilde{R}(n, n) t-\tilde{k}^{2} \tilde{R}(n, t) n .
\end{aligned}
$$

After some straightforward calculation, the above equation together with (3.3) and (2.18) we have

$$
\left(\tilde{\nabla}_{t} \tilde{R}\right)\left(\tilde{\nabla}_{t} t, t\right) t=\tilde{k}\left(\frac{r}{2}+3 f^{2}+2 f^{\prime}\right)^{\prime} n+\tilde{k} \tilde{\tau}\left(\frac{r}{2}+3 f^{2}+2 f^{\prime}\right) \xi .
$$

Applying $\phi^{2}$ in both sides of the above equation, we have

$$
\phi^{2}\left(\tilde{\nabla}_{t} \tilde{R}\right)\left(\tilde{\nabla}_{t} t, t\right) t=-\tilde{k}\left(\frac{r}{2}+3 f^{2}+2 f^{\prime}\right)^{\prime} n-\tilde{k} \tilde{\tau}\left(\frac{r}{2}+3 f^{2}+2 f^{\prime}\right) \xi .
$$

By virtue of (4.2) and the above relation, the theorem follows. 


\section{Slant curves in a three-dimensional f-Kenmotsu manifold with respect to Schouten-Van Kampen connection}

Definition 5.1. A unit speed curve $\gamma$ in an almost contact metric manifold $M(\phi, \xi, \eta, g)$ is said to be slant if its tangent vector field makes constant angle $\theta$ with $\xi$ i.e., $\eta(\dot{\gamma})=\cos \theta$ is constant along $\gamma$. By definition, slant curves with constant angle $\frac{\pi}{2}$ are called Legendre curves or almost contact curves. Slant curves in 3-dimensional Kenmotsu manifolds with respect to semi-symmetric metric connection have been studied by W. Tang, P. Majhi, P. Zhao and U.C. De [20]. In this section, we are interested to study slant curves on 3-dimensional f-Kenmotsu manifolds with respect to Schouten-Van Kampen connection. Let us consider a unit speed curve $\gamma$ on a f-Kenmotsu manifold, by virtue of (2.12) we get

$$
\tilde{\nabla}_{t} t=\nabla_{t} t+f(\xi-\eta(t) t)
$$

where $\nabla$ is Levi-Civita connection. Now if $\gamma$ is a Legendre curve in $\mathrm{M}$ and $\{t, n, b\}$ the Frenet frame along $\gamma$, then the tangent vector field $t$ can be defined by $\mathrm{t}=\dot{\gamma}$. Then from (5.1) we get

$$
\tilde{\nabla}_{t} t=\nabla_{t} t+f \xi
$$

Then from above equation we have:

Proposition 5.1. The curvature vector field $\nabla_{t} t$ coincides with the $\tilde{\nabla}_{t} t$ if and only if the manifold is cosymplectic.

Let $\gamma$ be a non-geodesic Frenet curve in three-dimensional f-Kenmotsu manifold with Schouten-Van Kampen connection.

Differentiating the equation $g(t, \xi)=\cos \theta$ with respect to $\mathrm{t}$ along $\gamma$ we get

$$
\tilde{\nabla}_{t} g(t, \xi)-g\left(\tilde{\nabla}_{t} t, \xi\right)-g\left(t, \tilde{\nabla}_{t} \xi\right)=0 .
$$

Using (2.12) in the above equation we get,

$$
\tilde{\kappa} \eta(n)=-\sin \theta \cdot \theta^{\prime},
$$

where $\{t, n, b\}$ is Frenet frame with $t=\dot{\gamma}$. If $\gamma$ is slant curve, then above equation reduces to

$$
\tilde{\kappa} \eta(n)=0 .
$$

Therefore, from the above equation we can state the following proposition:

Proposition 5.2. A non-geodesic curve $\gamma$ in a three-dimensional f-Kenmotsu manifold with Schouten-Van Kampen connection is slant if and only if it satisfies $\eta(n)=0$.

Hence the reeb vector field $\xi$ can be written as follows $\xi=\cos \theta t \mp \sin \theta b$. This means that the reeb vector field is in the plane spanned by $t$ and $b$, namely $g(\xi, n)=0$. On the other hand, with respect to an adapted local orthonormal frame fields $X, \phi X$, 
$\xi$ of $M$ such that $\eta(X)=0$ we have the following equalities of the Frenet vector fields $t, n, b$ for some function $\lambda(s)$,

$$
\begin{gathered}
t=\sin \theta\{\cos \lambda X+\sin \lambda \phi X\}+\cos \theta \xi, \\
n=-\sin \lambda X+\cos \lambda \phi X, \\
b=\mp \cos \theta \cos \lambda X \mp \cos \theta \sin \lambda \phi X \pm \operatorname{cosec} \theta \xi .
\end{gathered}
$$

Differentiating the equation $g(\xi, n)=0$ along the slant curve $\gamma$ of $M$, it follows that

$$
g\left(\tilde{\nabla}_{t} n, \xi\right)+g\left(n, \tilde{\nabla}_{t} \xi\right)=0
$$

using (2.6) and (2.12) we get

$$
\kappa \cos \theta \pm \tau \sin \theta=0
$$

Hence we can state the following theorem:

Theorem 5.1. If a non-geodesic curve of a three-dimensional f-Kenmotsu manifold with respect to Schouten-Van Kampen connection is a slant curve, then $\frac{\kappa}{\tau}=$ constant.

\section{An example of a three-dimensional f-Kenmotsu manifold with respect to Schouten-Van Kampen connection}

In this section, we would like to construct an example of a three-dimensional fKenmotsu manifold with respect to Schouten-Van Kampen connection.

We considered a three-dimensional manifold $M=\left\{(x, y, z) \in R^{3}, z \neq 0\right\}$, where $(x, y, z)$ are the standard coordinates in $R^{3}$. The vector fields

$$
e_{1}=z^{2} \frac{\partial}{\partial x}, \quad e_{2}=z^{2} \frac{\partial}{\partial y}, \quad e_{3}=\frac{\partial}{\partial z}
$$

are linearly independent at each point of $M$. Let $g$ be the Riemannian metric defined by

$$
g\left(e_{1}, e_{3}\right)=g\left(e_{2}, e_{3}\right)=g\left(e_{1}, e_{2}\right)=0, \quad g\left(e_{1}, e_{1}\right)=g\left(e_{2}, e_{2}\right)=g\left(e_{3}, e_{3}\right)=1 .
$$

Let $\eta$ be the 1 -form defined by $\eta(Z)=g\left(Z, e_{3}\right)$ for any $Z \in \chi(M)$. Let $\phi$ be the $(1,1)$ tensor field defined by $\phi\left(e_{1}\right)=-e_{2}, \phi\left(e_{2}\right)=e_{1}, \phi\left(e_{3}\right)=0$. Then using the linearity of $\phi$ and $g$ we have

$$
\eta\left(e_{3}\right)=1, \quad \phi^{2} Z=-Z+\eta(Z) e_{3}, \quad g(\phi Z, \phi W)=g(Z, W)-\eta(Z) \eta(W),
$$

for any $Z, W \in \chi(M)$. Thus for $e_{3}=\xi,(\phi, \xi, \eta, g)$ defines an almost contact metric structure on $M$. Now, by direct computations we obtain

$$
\left[e_{1}, e_{2}\right]=0, \quad\left[e_{2}, e_{3}\right]=-\frac{2}{z} e_{2}, \quad\left[e_{1}, e_{3}\right]=-\frac{2}{z} e_{1} .
$$


By Koszul formula

$$
\begin{array}{llll}
\nabla_{e_{1}} e_{3} & =-\frac{2}{z} e_{1}, & \nabla_{e_{1} e_{2}}=0, & \nabla_{e_{1}} e_{1}=-\frac{2}{z} e_{3}, \\
\nabla_{e_{2}} e_{3}=-\frac{2}{z} e_{2}, & \nabla_{e_{2}} e_{2}=-\frac{2}{z} e_{3}, & \nabla_{e_{2}} e_{1}=0, \\
\nabla_{e_{3}} e_{3}=0, & \nabla_{e_{3}} e_{2}=0, & \nabla_{e_{3}} e_{1}=0 .
\end{array}
$$

From above we see that the manifold satisfies $\nabla_{X} \xi=f(X-\eta(X) \xi)$ for $\xi=e_{3}$, where $f=-\frac{2}{z}$. Hence the manifold is a f-Kenmotsu manifold. Now the Schouten-Van Kampen connection on the manifold we have

$$
\begin{array}{rlrl}
\tilde{\nabla}_{e_{1}} e_{3} & =\left(-\frac{2}{z}-r\right) e_{1}, & \tilde{\nabla}_{e_{1} e_{2}}=0, & \tilde{\nabla}_{e_{1} e_{1}}=\frac{2}{z}\left(e_{3}-\xi\right), \\
\tilde{\nabla}_{e_{2}} e_{3} & =\left(-\frac{2}{z}-f\right) e_{2}, & \tilde{\nabla}_{e_{2}} e_{2}=\frac{2}{z}\left(e_{3}-\xi\right), & \tilde{\nabla}_{e_{2}} e_{1}=0, \\
\tilde{\nabla}_{e_{3}} e_{3}=-f\left(e_{3}-\xi\right), & \tilde{\nabla}_{e_{3}} e_{2}=0, & \tilde{\nabla}_{e_{3}} e_{1}=0 .
\end{array}
$$

From above we see that $\tilde{\nabla}_{e_{i}} e_{j}=0,(0 \leq i, j \leq 3)$ for $\xi=e_{3}$ and $f=-\frac{2}{z}$. Hence the manifold is f-Kenmotsu manifold with respect to Schouten-Van Kampen connection.

Example 6.1. Consider a curve $\gamma: I \rightarrow M$ defined by $\gamma(s)=\left(\sqrt{\frac{2}{3}} s, \sqrt{\frac{1}{3}} s, 1\right)$. Hence $\dot{\gamma}_{1}=\sqrt{\frac{2}{3}}$, where $\dot{\gamma}_{2}=\sqrt{\frac{1}{3}}$ and $\dot{\gamma}_{3}=0, \gamma(s)=\left(\gamma_{1}(s), \gamma_{2}(s), \gamma_{3}(s)\right)$. Now

$$
\begin{aligned}
\eta(\dot{\gamma}) & =g\left(\dot{\gamma}, e_{3}\right)=g\left(\dot{\gamma}_{1} e_{1}+\dot{\gamma}_{2} e_{2}+\dot{\gamma}_{3} e_{3}, e_{3}\right)=0 . \\
g(\dot{\gamma}, \dot{\gamma}) & =g\left(\dot{\gamma}_{1} e_{1}+\dot{\gamma}_{2} e_{2}+\dot{\gamma}_{3} e_{3}, \dot{\gamma}_{1} e_{1}+\dot{\gamma}_{2} e_{2}+\dot{\gamma}_{3} e_{3}\right) \\
& =\dot{\gamma}_{1}^{2}+\dot{\gamma}_{2}^{2}+\dot{\gamma}_{3}^{2} \\
& =\dot{\gamma}_{1}^{2}+\dot{\gamma}_{2}^{2} \\
& =\frac{2}{3}+\frac{1}{3} \\
& =1 .
\end{aligned}
$$

Hence the curve is Legendre curve.

Acknowledgement. The author is thankful to the referee for his/her valuable suggestions for the improvement of the paper.

\section{REFEREN CES}

1. Blair, D. E., Riemannian geometry of contact and symplectic manifolds, Progress in Math., Vol. 203, Birkhäuser, Boston, 2002.

2. Baikoussis, C. and Blair, D. E., On Legendre curves in contact 3-manifolds, Geometry Dedicata, 49(1994), 135-142.

3. Bejancu, A. and Faran,H., Foliations and Geometric structures, Math. Appl. 580, Springer, Dordrecht,2006.

4. Cho, J. T. and Lee, J. E., Slant curves in contact pseudo-Hermitian manifolds, Bull. Austral. Math. Soc., 78(2008), 383-396. 
5. Inoguchi, J. and Lee, J. E., Affine biharmonic curves in 3-dimensional homogenious geometries, Mediterr. J. Math., 10(2013), 571-592.

6. Ianus, S., Some almost product structures on manifolds with linear connection, Kodai Math. Semin. Rep., 23(1971), 305-310.

7. Janssens, D. and Vanheck, L., Almost contact structures and curvature tensors, Kodai Math. J., 4(1981),1-27.

8. Kenmotsu,K., A class of almost contact Riemannian manifolds, Tohoku math. J., 24(1972), 93-103.

9. Kazan, A. and Karadağ, H. B., Trans-Sasakian manifolds with Schouten-Van Kampen connection, Ilirias J. of Math., 7(2018), 1-12.

10. Lee, J.E., On Legendre curves in contact pseudo-Hermitian 3-manifolds, Bull. Aust. Math. Soc., 81(2010), 156-164.

11. Olszak, Z., Locally conformal almost cosymplectic manifolds, Colloq. Math., 57(1989), 73-87.

12. Olszak,Z. and Rosca, R., Normal locally conformal almost cosymplectice manifolds, Publ. Math., 39(1991), 315-323.

13. Olszak, Z., The Schouten-Van Kampen affine connection adapted to an almost(para) contact metric structure, Publications Delinstitut Mathematique, 94(2013),31-42.

14. Sarkar, A., Mondal, A. and Biswas, D., Some curves on three-dimensional transSasakian manifolds with semi-symmetric metric connection, Palestine J. of Math., 5(2016), 195-203.

15. Sarkar, A. and Mondal, Ashis, Some curves on $\alpha$-Sasakian manifolds with indefinite metric, J. of Calcutta Math. Soci., 13(2017), 25-34.

16. Sarkar, A., Hui, S. K. and Sen. M., A study on Legendre curves in three dimensional trans-Sasakian manifold, Lobachevskii J. Math., 35(1)(2014), 11-18.

17. Schouten, J. and Kampen, E. van, Zur Einbettungs-und Krümmungsthorie nichtholonomer Gebilde, Math. Ann., 103(1930), 752-783.

18. Solov'ev, A. F., On the curvature of the connection induced on a hyperdistribution in a Riemannian space, Geom. Sb., 19(1978), 12-23(in Russian).

19. Takahashi, T., Sasakian $\phi$-symmetric spaces, Tohoku Math J., 29(1977), 91-113.

20. Tang, W., Majhi, P., Zhao, P. and De, U. C., Legendre curves on 3-dimensional Kenmotsu manifolds admitting semisymmetric metric connection, Faculty of Sci. and Math, 10(2018), 3651-3656.

21. Yildiz, A., f-Kenmotsu manifolds with the Schouten-Van Kampen connection, Pub. De L'institut Math., 102(116)(2017), 93-105.

22. Welyczko, J., On Legendre curves in 3-dimensional normal almost contact metric manifolds, Soochow J. Math., 33(2007), 929-937.

\author{
Ashis Mondal \\ Department of Mathematics \\ Jangipur College \\ Murshidabad, Pin-742235 \\ West Bengal, India \\ ashism750@gmail.com
}

